\title{
Protocolo para la prevención de arritmias ventriculares debido al tratamiento en pacientes con COVID-19
}

\author{
Protocol for the prevention of ventricular arrhythmias \\ due to treatment in COVID-19 patients
}

Ángel Cueva-Parra,* Diego Neach-De la Vega, ${ }^{*}$ William Ortiz-Solís,* José Fernández-Domenech,* Selene Lara-Aguilera,* Sandra Chi-Pool,* Guillermo Muñoz-Benavides, ${ }^{*}$ Gabriela Bustillos-García,* Manlio Márquez-Murillo,* Jorge Gómez-Flores, Moisés Levinstein-Jacinto, ${ }^{*}$ Celso Mendoza-G, ${ }^{*}$ Santiago Nava-Townsend*

\section{JUSTIFICACIÓN}

n la actualidad se están haciendo muchos Eensayos clínicos en pacientes con enfermedad por coronavirus 2019 (COVID-19), con la intención de probar fármacos que sean útiles para su manejo. ${ }^{1}$ Algunos de éstos se asocian con la prolongación del tiempo de repolarización ventricular cardiaca (evidenciado en el electrocardiograma convencional como prolongación del intervalo QT), ello ocurre por alteración de diversos canales iónicos de la membrana celular en los miocitos cardiacos. ${ }^{2,3}$

La prolongación del intervalo QT predispone a la aparición de arritmias ventriculares que pueden causar la muerte. La arritmia que está más relacionada con la prolongación del intervalo QT es la taquicardia ventricular helicoidal o torsión de puntas, que puede degenerar rápidamente en fibrilación ventricular. ${ }^{4}$ Por otro lado, es importante mencionar que por cada 10 ms de prolongación del intervalo QT, el riesgo de torsión de puntas incrementa $7 \% .{ }^{4}$

Los pacientes con COVID-19 podrían Ilegar eventualmente a desarrollar síndrome de dificultad respiratoria aguda, la cual requerirá ventilación mecánica y manejo en una unidad de cuidados intensivos (UCI). Debido a su estado crítico, alteraciones electrolíticas secundarias a ese estado y a los múltiples medicamentos empleados, los pacientes en $\mathrm{UCl}$ pueden presentar QT prolongado. Se ha reportado que hasta $28 \%$ de los pacientes admitidos en una $\mathrm{UCI}$ tienen intervalo QT prolongado, y que uno de cada cinco pacientes tiene intervalo QT corregido (QTC) mayor de $500 \mathrm{~ms}$ al momento de su admisión en la $\mathrm{UCl}^{5}$

Algunos de los fármacos que están demostrando utilidad en el manejo de pacientes con COVID-19 son la hidroxicloroquina y la azitromicina; ambas pueden prolongar el intervalo QT y predisponer a arritmias malignas. ${ }^{2,3} \mathrm{Si}$ bien el riesgo de prolongar el QT tras el uso de estos medicamentos es bajo (alrededor de $1 \%)$, el número de pacientes con COVID-19 se eleva día a día; actualmente, la cifra mundial de casos supera los 400,000, si todos ellos recibieran estos medicamentos, se esperaría que al menos 4,000 pacientes prolonguen el QT. ${ }^{2}$ Por todo lo expuesto anteriormente y en el contexto de la epidemia mundial de COVID-19 que también está azotando México, nuestro servicio ha elaborado este protocolo.

\section{FUNDAMENTOS TEÓRICOS}

\section{El intervalo QT y el riesgo para arritmias ventriculares}

El intervalo QT es la representación electrocardiográfica, tanto de la despolarización como de la repolarización de los miocitos cardiacos. Este intervalo puede variar a lo largo del día y también experimenta cambios en función de 
la frecuencia cardiaca. ${ }^{6,7}$ Se han elaborado múltiples fórmulas para corregir el intervalo QT en función de la frecuencia cardiaca. En 1920, Bazett describió un método práctico para poder determinar el $\mathrm{QT}_{\mathrm{C}^{\prime}}$ denominado fórmula de Bazett:

$$
\text { QT corregido }=\frac{\text { QT medido }}{\sqrt{\text { Intervalo RR }}}
$$

El intervalo QT puede ser prolongado por múltiples medicamentos que generan directa o indirectamente inhibición de los canales iónicos de potasio $\left(I_{k}\right)$. La inhibición de éstos traerá consigo una prolongación anormal de la fase 3 del potencial de acción, lo que se traduce electrocardiográficamente como alteraciones de la onda $\mathrm{T}$ o aparición de la onda U. La prolongación de la fase 3 puede conllevar postdespolarizaciones tempranas, debido a la activación de canales de calcio que permiten el ingreso de éste dentro de la célula; esto último es lo que genera arritmias ventriculares, fundamentalmente torsión de puntas. ${ }^{4}$

Existen pacientes con alteraciones de canales iónicos debido a mutaciones genéticas que condicionan una prolongación patológica del intervalo QT, esta condición se denomina síndrome de QT largo. Actualmente, existen múltiples tipos de este síndrome y es una de las patologías hereditarias que se asocian con mayor frecuencia a muerte súbita. Se debe tener mucha precaución con los fármacos que se prescriben a estos pacientes, ya que podrían prolongar aún más el intervalo QT y precipitar arritmias malignas. ${ }^{7}$ Por el momento, no hay reportes de casos con síndrome de QT largo y COVID-19, pero si se presentara alguno, habrá que tener mucha precaución con los fármacos que se empleen. Incidencia de QT largo y posibilidad de esto en infecciones masivas COVID.

\section{Medición correcta del intervalo QT}

Es muy importante realizar una buena medición del intervalo QT. Uno de los errores más frecuentes al momento de aplicar la fórmula de Bazett es no colocar el intervalo RR en segundos. En la Figura 1 se muestra un ejemplo de la medición correcta del intervalo QT; en este ejemplo, el paciente tiene una frecuencia cardiaca de 63 latidos por minuto y, por consiguiente, un intervalo RR de 960 ms, que transformado a segundos serían 0.96 segundos. $^{8}$

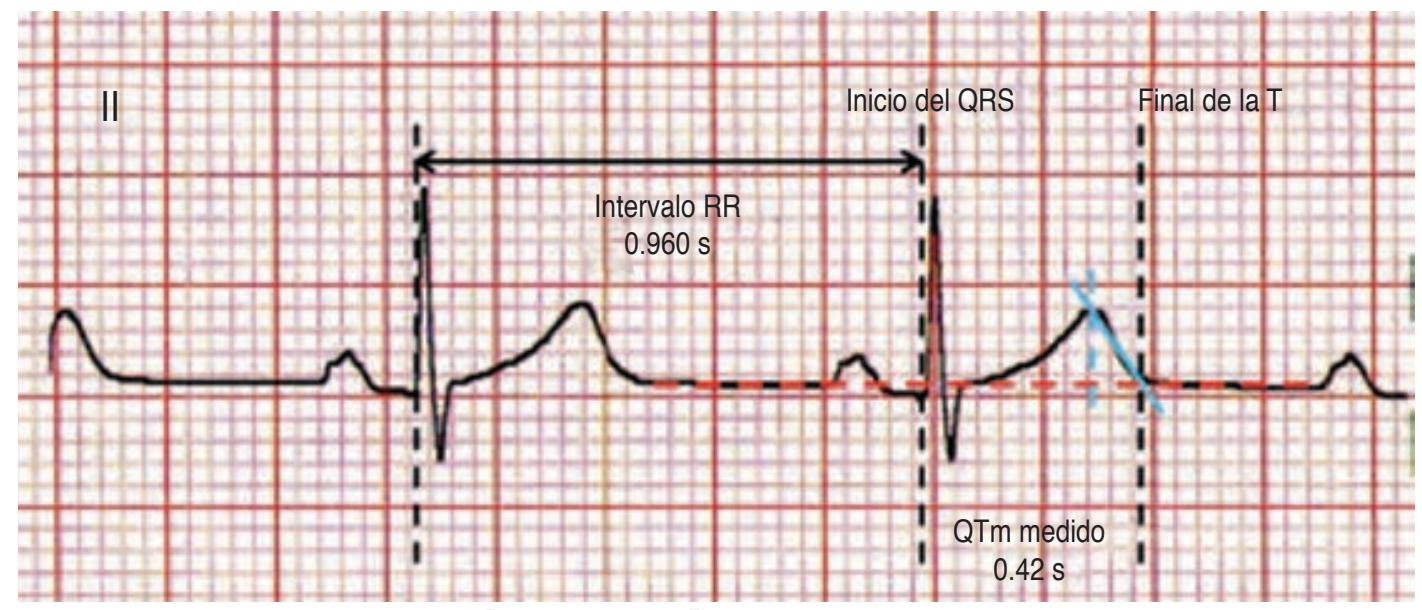

- El intervalo QT se mide desde el inicio del QRS hasta el final de la T (QTm)

Figura 1: inscripción descendente (línea azul completa) hasta donde

Medición correcta del intervalo QT

- Para determinar el final de la onda T se traza una línea desde su vértice (línea azul punteada), siguiendo la pendiente de su se cruza con la línea de base (línea punteada en rojo)

Corrección del QT con fórmula de Bazett:

$\frac{\mathrm{QTm}}{\sqrt{\mathrm{RR}}} \frac{0.42}{\sqrt{0.96}} \Longrightarrow \frac{0.42}{0.9797}=0.428$

Idealmente, se debe medir el QT en derivaciones con onda Q o DII y VS 
Finalmente, hay que mencionar que el intervalo QT debe medirse en las derivadas precordiales, idealmente en la derivada $\mathrm{V} 5 .^{8}$

\section{Hidroxicloroquina y azitromicina para pacientes con COVID-19}

Recientemente en Francia, Philippe Gautret y equipo llevaron a cabo un ensayo clínico en pacientes con COVID-19, en el cual emplearon hidroxicloroquina y azitromicina, basados en sus propiedades inmunomoduladoras demostradas previamente en estudios in vitro. Se encontró que la asociación hidroxicloroquinaazitromicina era útil para disminuir la carga viral en pacientes con COVID-19. Por lo tanto, el empleo de estos fármacos es una alternativa razonable para el manejo de la enfermedad. Cabe señalar que la duración del tratamiento fue de siete días y que se excluyeron pacientes con alergia a la hidroxicloroquina, con retinopatía y con QT prolongado. ${ }^{3}$

La hidroxicloroquina es un fármaco con muchas aplicaciones clínicas, fundamentalmente en la malaria y enfermedades reumáticas. Uno de sus efectos adversos es la inhibición del canal de potasio $\mathrm{KCNH} 2$ (el mismo que está afectado en el síndrome de QT largo tipo 2) y, por ende, puede prolongar el intervalo QT. ${ }^{2}$

La azitromicina es un macrólido de amplio espectro muy útil, no sólo por sus propiedades bactericidas, sino también inmunomodulado-

Tabla 1: Sistema de puntuación para predecir prolongación del intervalo QTc por fármacos (puntuación Tisdale).

Factores de riesgo

Puntuación

Edad $\geq 68$ años 1

Sexo femenino

Empleo de diuréticos de asa

1

Potasio sérico $\leq 3.5 \mathrm{mEq} / \mathrm{L}$

1

QTc al momento de la admisión $\geq 450 \mathrm{~ms}$

Infarto agudo de miocardio reciente

Uso de un fármaco que prolonga el QT

Uso de dos o más fármacos que prolongan el QT

Sepsis

Insuficiencia cardiaca

Puntuación máxima ras en diversos tipos de infecciones. Entre sus efectos adversos destacan los gastrointestinales, toxicidad hepática y prolongación del intervalo QT. Un estudio demostró que la azitromicina era un factor de riesgo para prolongación severa del QT (definida como mayor o igual de 500 $\mathrm{ms})$, con una odds ratio de 1.43, fundamentalmente en adultos mayores. ${ }^{9}$

\section{Antivirales}

Otra de las estrategias en la actualidad es el uso de antivirales, dentro de los cuales destacan el lopinavir y el ritonavir. Estos fármacos tienen muchos efectos adversos e interacciones farmacológicas; uno de los efectos más reconocido es la prolongación del intervalo PR, que podría incluso llegar a bloqueo auriculoventricular (AV) de tercer grado, y del intervalo QT, que puede ocasionar torsión de puntas. Estas alteraciones de la conducción $A \bigvee$ y la prolongación del QT son más frecuentes en pacientes con alteraciones de la conducción de base y que toman otros fármacos que prolongan el QT. Por último, debido a que el lopinavir y el ritonavir inhiben la enzima del citocromo CYP3A4, pueden incrementar los efectos adversos de otros medicamentos, como por ejemplo los anticoagulantes orales rivaroxabán y apixabán, así como del antiagregante plaquetario ticagrelor. ${ }^{10}$

Otros fármacos antivirales como la ribavirina y el remdesivir también se encuentran en estudio y parecen tener un mejor perfil de seguridad y menor cantidad de interacciones farmacológicas. ${ }^{10}$

\section{Riesgo para QT prolongado inducido por} fármacos y riesgo de arritmias ventriculares

No todos los pacientes tienen prolongación evidente del intervalo QT tras el uso de diversos medicamentos, existe una respuesta individualizada en cada paciente. Por ello es importante estratificar el riesgo para poder determinar, de manera más precisa, quiénes podrían presentar una prolongación evidente del QT. ${ }^{5}$

En el año 2014, Tisdale validó un sistema de puntuación para determinar qué pacientes pueden presentar una prolongación significativa del intervalo QT tras el uso de ciertos medicamentos (Tabla 1). Fue empleado en 
pacientes en $\mathrm{UCl}$ y resultó útil en la prevención de la aparición del intervalo QT mayor o igual de $500 \mathrm{~ms}$ y/o para determinar un incremento de $60 \mathrm{~ms}$, respecto al intervalo QT basal. ${ }^{11,12}$

\section{Tabla 2: Riesgo según la puntuación Tisdale.}

Tipo de riesgo

Puntuación

$\begin{array}{cc}\text { Bajo } & 0 \text { a } 6 \\ \text { Medio } & 7 \text { a } 10 \\ \text { Alto } & 11 \text { a } 21\end{array}$

Existen puntos de corte de este score. El riesgo puede ser bajo (menor de 7 puntos), medio (de 7 a 10 puntos) o alto (mayor o igual de 11 puntos) (Tabla 2). El riesgo para presentar QTc mayor o igual de 500 ms o prolongación de 60 ms o más es de 15\% para el grupo de bajo riesgo, $37 \%$ para el de riesgo moderado y $73 \%$ para alto riesgo. ${ }^{11,12}$

Debido a la pandemia actual, recientemente Giudicessi y su grupo han elaborado un protocolo de manejo para pacientes con COVID-19, con la intención de evitar arritmias ventriculares secundarias a la prolongación del intervalo QT por el uso de fármacos para el

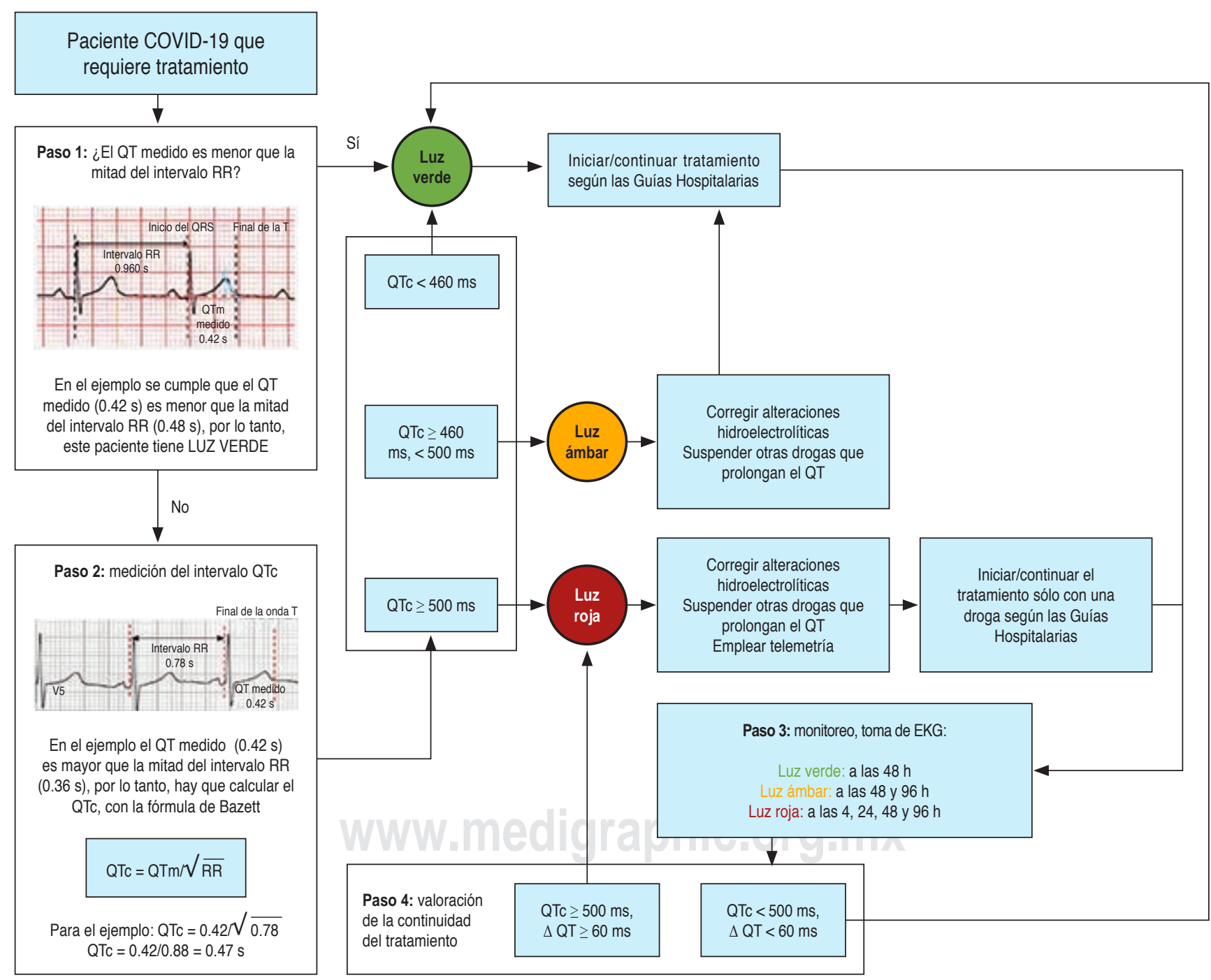

Figura 2: Algoritmo de manejo de los pacientes con COVID-19 que requieren tratamiento (adaptada de Giudicessi, et al., 2020). QTc = QT corregido; EKG = electrocardiograma. 
manejo de estos pacientes (Figura 2). ${ }^{2}$ La estratificación del riesgo se basa fundamentalmente en la medición del QT:

- Pacientes con QTc menor de 460 ms (semáforo con luz verde). Son el grupo de bajo riesgo para arritmias ventriculares y en ellos se puede iniciar el tratamiento con un fármaco o los dos.

- Pacientes con QTc mayor o igual de 460 ms, pero menor de 500 ms (semáforo con luz amarilla). Son el grupo de riesgo moderado para arritmias ventriculares; en ellos se deben vigilar estrechamente variables que alargan el QT, como el uso de otros fármacos, alteraciones electrolíticas, etc.

- Pacientes con QTc mayor o igual de 500 ms (semáforo con luz roja). Son el grupo de riesgo alto para arritmias ventriculares, en ellos debe usarse el tratamiento únicamente si el beneficio supera el riesgo.

Por otro lado, la forma más sencilla de valorar el riesgo y quizás un poco más práctica es considerar si el intervalo QT es menor de la mitad del intervalo RR precedente; si se cumple que sí lo es, el QTc siempre será menor de 460 $\mathrm{ms}$, lo cual indica que el paciente tiene luz verde para el inicio del tratamiento. ${ }^{2}$

Por el contrario, los pacientes con QTc basal mayor o igual a $500 \mathrm{~ms}$ antes del inicio del tratamiento sin la presencia de ninguna otra alteración de la conducción tienen luz roja, es decir, poseen riesgo alto de TV/F. En ellos se deberán descartar causas secundarias como alteraciones hidroelectrolíticas (hipopotasemia, hipocalcemia o hipomagnesemia) y descontinuar los fármacos innecesarios que pueden prolongar el QT, colocar telemetría, monitorizar de cerca y, si el beneficio del tratamiento supera el riesgo, considerar iniciar solo hidroxicloroquina y esperar con la azitromicina. ${ }^{2}$

Por último, si la terapia combinada con hidroxicloroquina y azitromicina que se inició en un paciente con luz verde prolonga el QT más de 60 ms, entonces se estará clasificando como un reactor de QT. El caso se deberá evaluar y considerar si debe o no continuar con la azitromicina y mejorar todas las variables que puedan prolongar el QT, como se comentó anteriormente. ${ }^{2}$

\section{MANEJO DE LOS PACIENTES}

Una vez identificado al paciente con $\mathrm{CO}$ VID-19 y con indicación de recibir hidroxicloroquina y/o azitromicina, se deben seguir los siguientes pasos previo al inicio de estos fármacos, para determinar si pueden recibirlos o no, descartando obviamente a los pacientes con síndrome de QT largo.

Primer paso: determinar si el intervalo QT medido es menor de la mitad del intervalo RR. Para este paso se deberá formular la siguiente pregunta: iel QT medido es menor de la mitad del intervalo RR? Si la respuesta es «sí», este paciente tendrá luz verde para iniciar el tratamiento que se crea conveniente (hidroxicloroquina, azitromicina o antivirales) y aquí concluye la evaluación para ellos. Si la respuesta es «no», se deberá pasar al siguiente paso, que es la estratificación del riesgo basado en la medición del QTC.

Segundo paso: estratificación del riesgo para el inicio del tratamiento.

1. Tomar un electrocardiograma de 12 derivadas, medir el intervalo QT en la derivada V5 y corregirlo con la fórmula de Bazett, así como niveles de electrolitos séricos $\left(\mathrm{K}^{+}, \mathrm{Mg}^{+2}, \mathrm{Ca}^{+2}\right)$.

2. Con las mediciones del QTC, determinar qué color de luz tiene nuestro paciente, según el semáforo anteriormente mostrado (véase la fórmula para corregir el QT en pacientes con QRS ancho).

a. Los pacientes con QTc menor de $460 \mathrm{~ms}$ poseen luz verde; es decir, pueden recibir los dos fármacos en combinación.

b. Los pacientes con QTC mayor o igual de 460, pero menor de $500 \mathrm{~ms}$ poseen luz ámbar; es decir, ellos podrían recibir uno o los dos fármacos en combinación, valorando la relación riesgo-beneficio, corrigiendo alteraciones hidroelectrolíticas si están presentes y suspendiendo otros fármacos que prolongan el QT y que no son necesarios al momento.

c. Los pacientes con QTc mayor o igual de 500 ms poseen luz roja; es decir, 
ellos sólo pueden recibir uno de los dos fármacos, valorando la relación entre riesgo y beneficio.

3. Otra alternativa válida puede ser aplicar el sistema de puntuación para predecir la prolongación del intervalo QTc por fárma$\cos$ (Tabla 1) y determinar el riesgo según la puntuación (Tabla 2), considerando todos los fármacos que se encuentra recibiendo el paciente. Los de bajo riesgo tendrán luz verde, los de riesgo moderado tendrán luz ámbar y los de alto riesgo luz roja.

Tercer paso: monitorización y seguimiento.

1. Monitoreo electrocardiográfico:

a. En los pacientes con luz verde, el siguiente control con electrocardiograma será a las 48 horas. No requieren otro electrocardiograma, salvo juicio clínico del médico tratante.

b. En los pacientes con luz ámbar, el siguiente control con electrocardiograma será a las 48 y luego a las 96 horas. No se requiere otro electrocardiograma, salvo juicio clínico del médico tratante.

c. En los pacientes con luz roja, el control con electrocardiograma será a las 4 horas de la primera dosis del medicamento, luego a las 24, 48 horas y a las 96 horas. No requieren otro electrocardiograma, salvo juicio clínico del médico tratante.

2. Monitoreo de electrolitos séricos:

a. En los pacientes con luz verde, el siguiente control de electrolitos séricos será a las 48 horas de iniciado el tratamiento y se corregirán de ser necesario. Posteriormente no se requieren controles, salvo juicio del médico tratante.

b. En los pacientes con luzámbar y roja, el siguiente control de electrolitos séricos será a las 24 horas de iniciado el tratamiento y se corregirán de ser necesario; el siguiente control será a las 96 horas de iniciado el tratamiento y se corregirá de ser necesario. Posteriormente no se requieren controles, salvo juicio del médico tratante.
Cuarto paso: valoración de la continuidad del tratamiento.

1. En pacientes que reciben hidroxicloroquina y/o azitromicina, deberá suspenderse primero la azitromicina sólo en los siguientes casos:

a. Presencia de taquicardia ventricular o fibrilación ventricular luego de iniciado el tratamiento.

b. Hallazgo de intervalo QTc $\geq 500 \mathrm{~ms}$ en los controles.

c. Incremento de $60 \mathrm{~ms}$ del intervalo QT con respecto al intervalo QT basal.

2. Posteriormente, valorar la necesidad de continuar o no la hidroxicloroquina, tomando en cuenta la relación entre riesgo y beneficio, lo cual queda a juicio del médico tratante. Además, es importante corregir todos los factores que puedan prolongar el QT.

3. De no cumplir las condiciones anteriormente mencionadas se puede continuar el tratamiento de manera segura.

\section{CONSIDERACIONES ESPECIALES}

\section{Pacientes portadores de dispositivos de estimulación cardiaca o QRS ancho}

Los pacientes portadores de dispositivos cardiacos o con alteraciones de la conducción intraventricular que generen QRS ancho (definido como mayor o igual de $120 \mathrm{~ms}$ ) tienden a tener una pseudoprolongación del intervalo QT. En este grupo se debe hacer el ajuste del intervalo QT, según la duración del QRS. ${ }^{2}$ La fórmula para corregir el QT en pacientes con QRS ancho es la siguiente:

$$
\begin{gathered}
\text { QTc ajustado para QRS } \\
\text { ancho=QTc-(QRS-100 ms) }
\end{gathered}
$$

Por ejemplo, en un paciente con bloqueo de rama izquierda con QRS de 180 ms y QTC en $540 \mathrm{~ms}$, impresionará que dicho intervalo está muy prolongado, pero al momento de corregirlo según la fórmula sería así: QTc ajustado para el QRS $=540-(180-100)$, dando como resultado final $460 \mathrm{~ms}$. 


\section{REFERENCIAS}

1. Clinical management of severe acute respiratory infection when COVID-19 disease is suspected [Internet]. World Health Organization; 2020 [consultado el 27 de marzo de 2020]. Disponible en: https://www. who. int/publications-detail/clinical-management-of-severeacute-respira- tory-infection-when-novel-coronavirus(ncov)-infection-is-suspected

2. Giudicessi JR, Noseworthy PA, Friedman PA, Ackerman MJ. Urgent guidance for navigating and circumventing the QTC prolonging and tor- sadogenic potential of possible pharmacotherapies for COVID-19. Mayo Clin Proc. 2020 Apr 7. doi: 10.1016/j. mayocp.2020.03.024. [Epub ahead of print]

3. Gautret P, Lagier JC, Parola P, Hoang VT, Meddeb L, Mailhe $M$, et al. Hydroxychloroquine and azithromycin as a treatment of COVID-19: results of an open-label non-randomized clinical trial [published online ahead of print, 2020 Mar 20]. Int J Antimicrob Agents. 2020; 105949.

4. Gupta A, Lawrence AT, Krishnan K, Kavinsky CJ, Trohman RG. Current concepts in the mechanisms and management of drug-induced QT prolongation and torsade de pointes. Am Heart J. 2007; 153 (6): 891-899.

5. Tisdale JE, Wroblewski HA, Overholser BR, Kingery JR, Trujillo TN, Kovacs RJ. Prevalence of QT interval prolongation in patients admitted to cardiac care units and frequency of subsequent administration of QT interval-prolonging drugs: a prospective, observational study in a large urban academic medical center in the US. Drug Saf. 2012; 35 (6): 459-470.

6. Bazett HC. An analysis of the time-relations of electrocardiograms. Heart. 1920; 7: 35-70.

7. Márquez MF. El síndrome de QT largo: una breve revisión del diagnóstico electrocardiográfico incluyendo la prueba de Viskin. Arch Cardiol Mex. 2012; 82 (3): 243-247.

8. Asensio E, Acunzo R, Uribe W, Saad EB, Sáenz L. Recomendaciones para la medición del intervalo QT durante el uso de medicamentos para el tratamiento de infección por COVID-19. Actualizables de acuerdo con la disponibilidad de nueva evidencia [Internet]. Sociedad Latinoamericana del Ritmo Cardiaco (LAHRS), Colegio Colombiano de Electrofisiología, Sociedad Argentina de Electrofisiología Cardiaca (SADEC), Sociedad Brasileña de Arritmias Cardiacas (SOBRAC), Sociedad Mexicana de Electrofisiología Cardiaca (SOMEEC). Disponible en: https://www. sociedadsadec.org.ar/wp-content/uploads/2020/04/ RECOMENDACIONES-PARA-LA-MEDICION-DELINTERVALO-QT-DURANTE-EL-USO-DE-MEDICAMENTOS-PARA-EL-TRATAMIENTO-DE-INFECCIONPOR-COVID.pdf

9. Choi Y, Lim HS, Chung D, Choi JG, Yoon D. Risk Evaluation of azithromycin-induced QT prolongation in real-world practice. Biomed Res Int. 2018; 2018: 1574806.

10. Driggin E, Madhavan MV, Bikdeli B, Chuich T, Laracy J, Bondi-Zoccai G et al. Cardiovascular considerations for patients, health care workers, and health systems during the coronavirus disease 2019 (COVID-19) pandemic. J Am Coll Cardiol. 2020 Mar 18. doi: 10.1016/j. jacc.2020.03.031. [Epub ahead of print]

11. Tisdale JE, Jaynes HA, Kingery JR, Mourad NA, Trujillo TN, Overholser BR et al. Development and validation of a risk score to predict QT interval prolongation in hospitalized patients. Circ Cardiovasc Qual Outcomes. 2013; 6 (4): 479-487.

12. Tisdale JE, Jaynes HA, Kingery JR, Overholser BR, Mourad NA, Trujillo TN et al. Effectiveness of a clinical decision support system for reducing the risk of QT interval prolongation in hospitalized patients. Circ Cardiovasc Qual Outcomes. 2014; 7 (3): 381-390.

\section{Correspondencia:}

Santiago Nava-Townsend

E-mail: santiagonavat@hotmail.com

\section{RESPONSABILIDADES ÉTICAS}

Protección de personas y animales. Los autores declaran que para esta investigación no se han realizado experimentos en seres humanos ni en animales.

Confidencialidad de los datos. Los autores declaran que han seguido los protocolos de su centro de trabajo sobre la publicación de datos de pacientes.

Derecho a la privacidad y consentimiento informado. Los autores han obtenido el consentimiento informado de los pacientes y/o sujetos referidos en el artículo. Este documento obra en poder del autor de correspondencia. 